\title{
Stellar CME activity and its possible influence on exoplanets' environments: Importance of magnetospheric protection
}

\author{
Maxim L. Khodachenko ${ }^{1,2}$, Yury Sasunov ${ }^{1}$, Oleksiy V. Arkhypov ${ }^{1}$, \\ Igor I. Alexeev ${ }^{2}$, Elena S. Belenkaya ${ }^{2}$, \\ Helmut Lammer ${ }^{1}$, Kristina G. Kislyakova ${ }^{1}$, \\ Petra Odert $^{3}$, Martin Leitzinger ${ }^{3}$, and Manuel Güdel ${ }^{4}$ \\ ${ }^{1}$ Space Research Institute, Austrian Academy of Sciences, \\ 8042 Graz, Austria \\ email: maxim.khodachenko@oeaw.ac.at \\ ${ }^{2}$ Institute of Nuclear Physics, Moscow State University, \\ 119992 Moscow, Russia \\ ${ }^{3}$ Institute of Physics, Karl-Franzens-University, \\ 8010 Graz, Austria \\ ${ }^{4}$ Institute of Astronomy, University of Vienna, \\ 1180 Vienna, Austria
}

\begin{abstract}
CMEs are large-scale magnetized plasma structures carrying billions of tons of material that erupt from a star and propagate in the stellar heliosphere, interacting in multiple ways with the stellar wind. Due to the high speed, intrinsic magnetic field and the increased plasma density compared to the stellar wind background, CMEs can produce strong effects on planetary environments when they collide with a planet. The main planetary impact factors of CMEs, are associated interplanetary shocks, energetic particles accelerated in the shock regions, and the magnetic field disturbances. All these factors should be taken into account during the study of evolutionary processes on exoplanets and their atmospheric and plasma environments. CME activity of a star may vary depending on stellar age, stellar spectral type and the orbital distance of a planet. Because of relatively short range of propagation of majority of CMEs, they impact most strongly the magnetospheres and atmospheres of close orbit $(<0.1 \mathrm{AU})$ exoplanets.
\end{abstract}

Keywords. magnetic fields, plasmas, stars:planetary systems, stars:winds, stars:activity

\section{Introduction}

The constantly growing number of discovered exoplanets and accumulation of data regarding their physical and orbital characteristics provide an empirical platform for a more detailed study of general principles and major trends of the formation and evolution of planets and planetary systems (including the planetary potential habitability aspect). More than a half of known exoplantes have orbits around their host stars shorter than 0.6 AU. By this, an evident maximum in the orbital distribution of exoplanets takes place in the vicinity of $0.05 \mathrm{AU}$, with two well pronounced major sub-populations there corresponding to the giant type planets $\left(0.2 M_{\mathrm{J}}<m_{\mathrm{p}}<8 M_{\mathrm{J}}\right)$, so called "Hot Jupiters", and less massive $\left(0.008 M_{\mathrm{J}}<m_{\mathrm{p}}<0.08 M_{\mathrm{J}}\right)$, Neptune- and Super-Earth type planets. Here $M_{\mathrm{J}}$ stays for the mass of Jupiter. Altogether the Hot Jupiters comprise about $30 \%$ of the total number of known exoplanets.

Close location of the majority of known exoplanes to their host stars results in intensive heating, ionization, and chemical modification of their upper atmospheres by the 
stellar X-ray/EUV (XUV) radiation with the subsequent expansion of the ionized atmospheric material and its loss due to interaction with the stellar wind (Lammer et al., 2009; Khodachenko et al., 2007a,b). A number of actual questions regarding the evolutionary paths of planetary systems and influencing them key factors is nowadays under continuous tackling. Among these questions a prominent position belongs to the problem of stellar - planetary interactions, including consideration of influences of stellar radiation and plasma flows, e.g., stellar wind, coronal mass ejections (CMEs), on planetary environments and evolution of planets. Magnetic fields, those connected with the planetary intrinsic magnetic dipole $\mathcal{M}$, as well as the magnetic fields associated with the electric current systems induced in the planetary close surroundings, play here an important role. They the planetary magnetosphere which appears as an obstacle (magnetospheric obstacle) interacting with a stellar wind and protecting the internal planetary environments (ionosphere, atmosphere, surface) against of direct impact of stellar plasmas and energetic particles (e.g., cosmic rays).

The plasma of stellar CMEs colliding with a planet, interacts with the planetary magnetosphere, and in the case of a weak magnetospheric protection (i.e., weak or no intrinsic planetary magnetic dipole), the magnetosphere is compressed down to the planetary surface, resulting in strong erosion of the planetary atmosphere. Sufficiently large magnetospheres are known to protect the underlying planetary environments, e.g. ionosphere, atmosphere, and surface against of stellar XUV/EUV and stellar wind factors. These usually require strong enough intrinsic planetary magnetic fields and/or extended magnetospheric current systems such as magnetodisks. Below we discuss the role of such factors like activity of a host star and intrinsic magnetic field of a planet and show how the account of these factors may influence the scaling of the planetary magnetosphere and its protecting capabilities.

\section{Impact of stellar radiation and plasma flows on planets}

Interaction of short-periodic exoplanets with the stellar wind plasma and high XUV flux at close orbital distances plays a crucial role regarding the ionization and ion loss processes of atmospheric species. The action of intensive stellar radiation and stellar winds on planetary environments consists of the following effects.

1) XUV radiation of the host star affects the the planetary thermosphere heat budget, resulting in the heating and expansion of the upper atmosphere, which under certain conditions could be so large that the majority of light atmospheric constituents overcome the gravitational binding and escape from the planet in the form of a hydrodynamic wind. This effect is called as a hydrodynamic or thermal escape (Tian et al., 2008; Penz et al., 2008; Erkaev et al., 2013). Simultaneously with the direct radiational heating of the upper atmosphere, the processes of ionization with the consequent production of energetic neutral atoms (ENAs) by various photo-chemical and charge exchange reactions take place (Lammer et al., 2008; Lichtenegger et al., 2009). Such processes, together with the thermal escape, result in the formation around planets of extended (in some cases) coronas, filled with hot neutral atoms.

2) The expanding upper planetary atmospheres and/or hot neutral coronas may reach and even exceed, the boundaries of the planetary magnetospheres. In this case they will be directly exposed to the plasma flows of the stellar wind and CMEs with the consequent loss due to ion pick-up, as well as sputtering, and different kinds of photo-chemical energizing mechanisms which all contribute to the so-called non-thermal atmospheric mass-loss process (Lichtenegger et al., 2009). As a crucial parameter here appears the 
size of the planetary magnetosphere. Altogether, this makes the planetary magnetic field, as well as the parameters of the stellar wind (mainly density $n_{\mathrm{sw}}$ and speed $v_{\mathrm{sw}}$ ) to be very important for the processes of atmospheric erosion and mass-loss of a planet, affecting finally the whole evolution of its environments. By this, the size of magnetosphere and its planetary protecting role should be always considered in context with the fact that the stellar radiation and plasma flows may vary significantly throughout the lifetime of the host star, as its luminosity and activity evolve. This evolution is different for different star types, and depends also on their age.

In that respect, the interaction of close-orbit exoplanets with stellar CMEs appears to be an important process, which is central to a better understanding of the non-thermal mass loss mechanism. High speed, intrinsic magnetic field and the increased density as compared to the stellar wind background, make CMEs an active factor which strongly influences the planetary environments and magnetospheres. Often collisions of the closeorbit exoplanets with massive stellar CME plasmas should compress planetary magnetospheres much deeper towards the surface of the exoplanet. This would result in much higher ion loss rates than that expected during the usual stellar wind conditions.

\subsection{Stellar activity}

The relevant physical phenomena of stellar activity on late-type stars (i.e., spectral classes G, K, M) and their observational manifestations include modulations of the stellar photospheric light due to stellar spots, intermittent and energetic flares, coronal mass ejections (CMEs), stellar cosmic rays, enhanced XUV emissions (see Scalo et al. (2007) and references therein). Evaluation of flaring rates and intensities usually require long-duration monitoring. So, the proxies for the flaring activity are used, such as optical Ca $\mathrm{H}$ and K emission cores, H-alpha and Mg II emission, soft X-ray continuous emission, and a large number of UV-to soft X-ray emission lines (Ayres 1997; Gershberg 2005).

Observations of stars in clusters have revealed that single late-type stars spin down monotonically with their age because of angular momentum loss (Skumanich 1972]). For a given age of star $t$, the stellar rotation period can be estimated as (Newkirk 1980): $P_{\text {rot }} \propto\left(1+\frac{t}{\tau}\right)^{0.7}$, where $\tau=2.56 \times 10^{7}$ yrs is a time constant calculated by Newkirk (1980)]. At the same time, already early studies pointed out a strong correlation between the rotation rate of a star and its activity level (Wilson 1966). This correlation means that there must be dependence between stellar activity and age. For solar-type stars this has been studied within the "Sun in Time" project (Ribas et al., 2005). Based on the analysis of a large amount of X-ray, EUV and UV observations of a homogeneous sample of single nearby G0-5 stars with known rotation periods, luminosity and ages, it has been concluded that during the fist 100 Myr after the Sun arrived at the ZeroAge Main-Sequence (ZAMS), the integrated XUV flux was up to 100 times higher than today. After this very active stage, XUV flux of a solar-type star decreases with the time: $\propto t[G y r]^{-1.72}$ (Ribas et al., 2005).

According to the currently accepted paradigm, the wide range of activity levels and related phenomena observed in different stars is directly connected with operation of the stellar magnetic dynamo. By this, two basic parameters: $(i)$ stellar rotation rate and (ii) depth of the convective zone, are believed to control the stellar dynamo efficiency, which increases with increasing of any, or both of these quantities. Since the stellar convective envelope becomes thicker with decreasing stellar mass, it is straightforward to infer that, at a given rotation period (i.e. age), the low-mass M- and K- stars should be more active than a solar-type G- star. This fact has many observational confirmations. For example, a relatively old ( 5.5 Gyr) dwarf M- star, Proxima Centauri, experiences measurable flares at a rate of about one flare per hour (Walker 1981). 


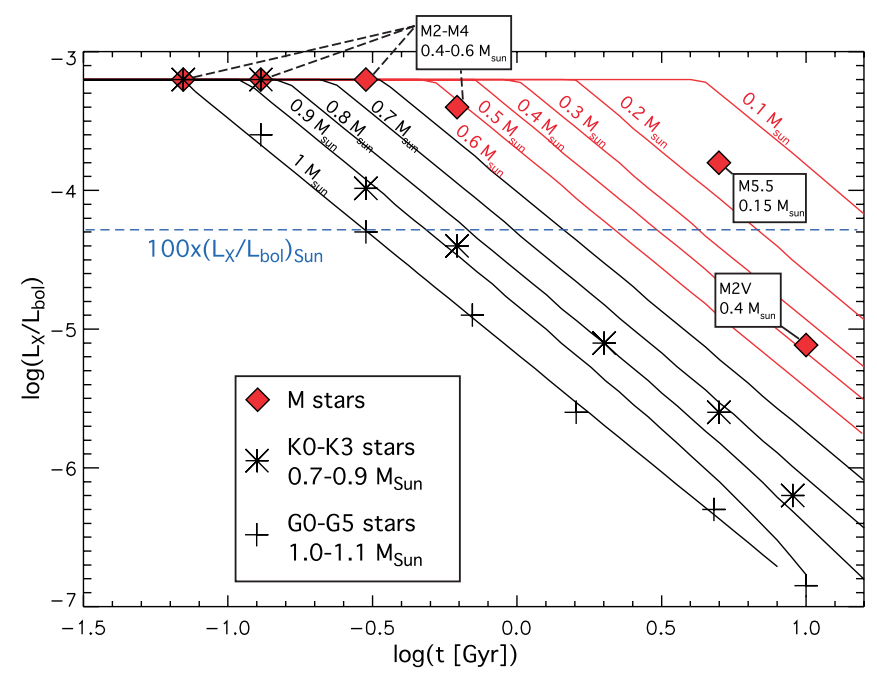

Figure 1. $L_{\mathrm{X}} / L_{\mathrm{bol}}$ as a function of age for stars with masses $<M_{\mathrm{Sun}}$. Symbols represent stars from the "Sun in Time" program (adopted from Scalo et al. (2007).

Audard et al., (2000) found that the energy of flares correlates with the stellar activity, characterized by $L_{\mathrm{X}} / L_{\mathrm{bol}}$, where $L_{\mathrm{X}}$ and $L_{\mathrm{bol}}$ are X-ray and bolometric luminosities of a star, respectively. The evolution of $\log \left(L_{\mathrm{X}} / L_{\mathrm{bol}}\right)$ with time for stars of various masses is shown in Figure 1, provided by Scalo et al. (2007). According to this activity-age diagram, the solar-type G- stars stay at saturated emission levels only until ages of $\sim 100 \mathrm{Myr}$, and then their XUV luminosities rapidly decrease with age: $\propto(t[G y r])^{-1.72}$. On the other hand, M- stars have saturated emission periods up to 0.5-1 Gyr, and then their luminosity decreases in a way similar to the solar-type stars. According to Audard et al., (2000), the rate of high-energy $\left(E>10^{32} \mathrm{erg}\right)$ flares per day as $\left.\log N\right|_{E>10^{32}} \mathrm{erg}=-26.7+0.95 \log L_{X}$, which in the case of M- stars with a saturated activity level $L_{X}=7 \times 10^{28} \mathrm{erg} / \mathrm{s}$ implies $\sim 6$ strong flares per day. Therefore, the powerful flares occur more often in X-ray bright stars. Altogether it has been found (Ribas et al., 2005; Scalo et al., 2007) that early Kstars and early M-stars may have XUV emissions level, and therefore flaring rates, of $\sim(3-4)$ and $\sim(10-100)$, respectively, times higher than solar-type G-stars of the same age.

\subsection{Stellar winds and CMEs}

In addition to being exposed to electromagnetic radiation from their host stars, exoplanets are also exposed to high-speed outflows of particles from the stellar atmosphere. For cool main sequence stars like the Sun, stellar winds arise in the hot coronas that represent the outermost atmospheres of the stars. Although the mechanisms of coronal heating and coronal wind acceleration remain hot topics of research, Parker (1958) demonstrated long ago that if once you have a hot corona, a wind much like that of the Sun arises naturally through thermal expansion. Thus, any star known to have a hot corona can be expected to possess a coronal wind. Observations from X-ray observatories such as Einstein, ROSAT, Chandra, and XMM-Newton have demonstrated that $\mathrm{X}$-ray emitting coronas are ubiquitous among cool main sequence stars, so coronal winds can be expected to be a common feature as well. Unfortunately, detecting and studying these winds is much harder than detecting and studying the coronas in which they arise. Current observational capabilities do not yet allow us to directly detect solar-like coronal winds emanating from other stars. 
Recently, there have been important developments towards indirect detections of stellar winds through their interactions with the surrounding interstellar medium. In particular, the stellar mass loss rates and related stellar wind parameters have been estimated by observing astrospheric absorption features of several nearby G- and K-stars. Comparison of the measured absorption to that calculated by hydrodynamic codes made it possible to perform empirical estimation of the evolution of the stellar mass loss rate as a function of stellar age (Wood et al., 2002; 2005) and to conclude about the dependence of $n_{\mathrm{sw}}$ and $v_{\mathrm{sw}}$ on the age of the stellar system. In particular, the younger solar-type G- stars appeared to have much denser and faster stellar winds as compared to the present Sun. Combining the stellar mass loss measurements of (Wood et al., 2005) with the results of (Newkirk, 1980) for the age-dependence of stellar wind velocity, Grießmeier et al. (2007) proposed a method for calculation of stellar wind density $n_{\mathrm{sw}}$ and velocity $v_{\mathrm{sw}}$ at a given orbital location of an exoplanet $d$ for a given mass $M_{*}$, radius $R_{*}$ and age $t_{*}$ of a star. As an example, the values of stellar wind plasma parameters for a solar-analog G-type star $\left(M_{*}=M_{\text {Sun }}\right.$, age: $\left.4 \mathrm{Gyr}\right)$ at orbital distances of $0.045 \mathrm{AU}, 0.1 \mathrm{AU}$, and $0.3 \mathrm{AU}$ are given in Table 1.

Furthermore, it is known from observations of our Sun that flaring activity of a star is accompanied by eruptions of coronal mass (e.g. CMEs), occurring sporadically and propagating in the stellar wind as large-scale plasma-magnetic structures. Traveling outward from the star at high speeds (up to thousands $\mathrm{km} / \mathrm{s}$ ), CMEs create major disturbances in the interplanetary medium and produce strong impacts on the planetary environments and magnetospheres. Since CMEs can be directly observed only on the Sun, the current knowledge on them comes from the study of the Sun and the heliosphere. On the Sun, CMEs are associated with flares and prominence eruptions and their sources are usually located in active regions and prominence sites. The likelihood of CME-events increases with the size and power of the related flare event. Generally, it is expected that the frequent and powerful flares on magnetically active flaring stars should be accompanied by an increased rate of CME production. By considering the Sun as a typical representative of G- stars, it seems reasonable to assume a similarity of the basic parameters of the stellar winds of G- stars and those known for the Sun. Such a solar-stellar analogy principle is widely considered for the investigation of basic processes of the stellar wind - planet interaction. Based on the estimations of solar CME plasma density $n_{\mathrm{CME}}$, using the in-situ spacecraft measurements (at distances $>0.4 \mathrm{AU}$ ) and the analysis of white-light coronagraph images (at distances $\leqslant 30 R_{\text {Sun }} \approx 0.14 \mathrm{AU}$ ), Khodachenko et al. (2007a) provided general power-law interpolations of $n_{C M E}$ dependence on the distance to a star:

$$
n_{\mathrm{CME}}^{\min }(d)=4.88(d[\mathrm{AU}])^{-2.3}, \quad n_{\mathrm{CME}}^{\max }(d)=7.10(d[\mathrm{AU}])^{-3.0},
$$

Equations (2.1) identify a typical maximum-minimum range of $n_{\mathrm{CME}}$. The dependence of stellar CME speed $v_{\mathrm{CME}}$ on the orbital distance $d$ can be approximated by the formula:

$$
v_{\mathrm{CME}}=v_{0}\left(1-e^{\frac{2.8 R_{\mathrm{Sun}}-d}{8 \cdot 1 R_{\mathrm{Sun}}}}\right)^{1 / 2},
$$

proposed in Sheeley et al. (1997) on the basis of tracking of several solar wind density enhancements at close distances $(d<0.1 \mathrm{AU})$. For the approximation of average- and high- speed CMEs one may take in $(2.2) v_{0}=500 \mathrm{~km} / \mathrm{s}$ and $v_{0}=800 \mathrm{~km} / \mathrm{s}$, respectively. Besides of that, the average mass of CMEs is estimated as $10^{15} \mathrm{~g}$, whereas their average duration at distances $\sim 0.05 \mathrm{AU}$ is close to 8 hours. Table 1 provides an example of stellar CME plasma parameters for a solar-analog G-type star at orbital distances of $0.045 \mathrm{AU}$, $0.1 \mathrm{AU}$, and 0.3 AU. Because of the relatively short range of propagation of majority 
Table 1. Stellar wind and CME parameters for a solar-analog G-type star $\left(M_{*}=M_{\mathrm{Sun}}\right.$, age: 4 Gyr) at different orbital distances. The values of $\tilde{v}_{\mathrm{CME}}^{f a s t}$ and $\tilde{v}_{\mathrm{CME}}^{a v}$ are obtained using (2.2) with $v_{0}=800 \mathrm{~km} / \mathrm{s}$ and $v_{0}=500 \mathrm{~km} / \mathrm{s}$, respectively. All the velocities include a contribution of the Keplerian planetary orbital velocity $V_{K}$.

\begin{tabular}{lcccc}
\hline Orbital distance & $\begin{array}{c}n_{\mathrm{sw}} \\
{\left[\mathrm{cm}^{-3}\right]}\end{array}$ & $\begin{array}{c}\tilde{v}_{\mathrm{sw}} \\
{[\mathrm{km} / \mathrm{s}]}\end{array}$ & $\begin{array}{c}n_{\mathrm{CME}}^{\min } / n_{\mathrm{CME}}^{\max }\left[\mathrm{cm}^{-3}\right] \\
\tilde{v}_{\mathrm{CME}}^{a v} / \tilde{v}_{\mathrm{CME}}^{\text {fast }} \\
{[\mathrm{km} / \mathrm{s}]}\end{array}$ \\
\hline $0.045 \mathrm{AU}$ & $9.1 e 3$ & 210 & $6.1 e 3 / 7.8 e 4$ & $520 / 810$ \\
$0.1 \mathrm{AU}$ & $1.2 e 3$ & 260 & $\begin{array}{c}1.0 e 3 / 7.1 e 3 \\
78 / 2.6 e 2\end{array}$ & $510 / 810$ \\
$0.3 \mathrm{AU}$ & 92 & 340 & $780 / 800$ \\
\hline
\end{tabular}

of CMEs, they should strongly impact first of all the planets at close orbits $(\lesssim 0.3$ AU). Khodachenko et al. (2007a) have found that for a critical CME production rate $f_{\mathrm{CME}}^{c r} \approx 36$ CMEs per day (and higher) a close orbit exoplanet appears under continuous action of the stellar CMEs plasma, so that each next CME collides with the planet during the time when the previous CME is still passing over it. This means in general the harder conditions for the planetary environments than those in the case of a regular stellar wind. Therefore, the investigation of evolutionary paths of close-orbit exoplanets in potentially habitable zones around young active stars, besides of the higher XUV radiation, should take also into account the effects of "short range" (in astrophysical scales) planetary impacting factors of stellar activity such as relatively dense stellar winds and frequent magnetic clouds (MCs) and CMEs.

\section{The problem of magnetospheric protection of exoplanets}

For an efficient magnetospheric protection of a planet, the size of its magnetosphere characterized by the magnetopause stand-off distance $R_{\mathrm{S}}$ should be much larger than the height of the exobase. By this, the value of $R_{\mathrm{s}}$ is determined from the balance between the stellar wind ram pressure and the planetary magnetic field pressure at the substellar point (Grießmeier et al., 2004; Khodachenko et al., 2007a). In the most of studies so far, the investigation of an exoplanetary magnetospheric protection is performed within a highly simplifying assumption of a planetary dipole-dominated magnetosphere. This means that only the intrinsic magnetic dipole moment of an exoplanet $\mathcal{M}$ and the corresponding magnetopause electric currents (i.e., "screened magnetic dipole" case) are considered as the major magnetosphere forming factors. In this case, i.e. assuming $B(r) \propto \mathcal{M} / r^{3}$, the value of $R_{\mathrm{s}}$ has been defined by the following expression:

$$
R_{\mathrm{s}} \equiv R_{\mathrm{s}}^{(d i p)}=\left[\frac{\mu_{0} f_{0}^{2} \mathcal{M}^{2}}{8 \pi^{2} \rho_{\mathrm{sw}} \tilde{v}_{\mathrm{sw}}^{2}}\right]^{1 / 6}
$$

where $\mu_{0}$ is the diamagnetic permeability of free space, $f_{0} \approx 1.22$ is a form-factor of the magnetosphere caused by the account of the magnetopause electric currents, $\rho_{\mathrm{sw}}=n_{\mathrm{sw}} m$ is the mass density of the stellar wind, and $\tilde{v}_{\mathrm{sw}}$ is the relative velocity of the stellar wind plasma which includes also the planetary orbital rotation velocity. For the tidally locked close orbit exoplanets with weak magnetic moments exposed to a dense and/or fast stellar wind plasma flows, (3.1) yields rather small values for sizes of dipole-dominated magnetospheres, $R_{\mathrm{s}}=R_{\mathrm{s}}^{(d i p)}$, compressed by the stellar wind plasma flow, which in the most extreme cases may even shrink down to the planetary radius $r_{\mathrm{p}}$. Therefore, the approach to estimation of the magnetosphere size based on (3.1) resulted in the commonly 
accepted conclusion, that in order to have an efficient magnetic shield, a planet needs a strong intrinsic magnetic dipole $\mathcal{M}$.

Khodachenko et al. (2007b) studied the mass loss of the Hot Jupiter HD 209458b due to the ion pick-up mechanism caused by stellar CMEs, colliding with the planet. In spite of the sporadic character of the CME-planetary collisions, in the case of a moderately active host star of HD 209458b, it has been shown that the integral action of the stellar CME impacts over the exoplanet's lifetime can produce significant effect on the planetary mass loss. The estimates of the non-thermal mass loss of the weakly magnetically protected Hot Jupiter, HD 209458b, due the stellar wind and CMEs ion pick-up, lead to significant and sometimes unrealistic values - up to several tens of planetary masses $M_{\mathrm{p}}$ lost during a planet life time (Khodachenko et al., 2007b). In view of the fact that multiple closein giant exoplanets, comparable in mass and size with the Solar System Jupiter exist, and that it is unlikely that all of them began their life as ten times, or even more massive objects, one may conclude that additional factors and processes have to be taken into consideration in order to explain the protection of close-in exoplanets against of destructive non-thermal mass loss. In the following sub-section we introduce a more complete model of magnetosphere of a giant gas exoplanet, which due to its consequent account of the specifics of close orbit Hot Jupiters provides under similar conditions larger sizes for the planetary magnetospheric obstacles, then those given by the simple screened magnetic dipole model, traditionally considered so far in the literature.

\section{Magnetodisk-dominated magnetosphere of a Hot Jupiter}

The investigation of exoplanetary magnetospheres and their role in evolution of planetary systems forms a new and fast developing branch. Magnetosphere of a close orbit exoplanet is a complex object, which formation depends on different external and internal factors. These factors may be subdivided on two basic groups: (a) stellar factors, e.g., stellar radiation, stellar wind plasma flow, stellar magnetic field and (b) planetary factors, e.g., type of planet, orbital characteristics, escaping material flow, and planetary magnetic field. The structure of an exoplanetary magnetosphere depends also on the speed regime of the stellar wind plasma relative the planet (Erkaev et al., 2005; Ip et al., 2004). In particular, for an exoplanet at sufficiently large orbital distance when the stellar wind is super-sonic and super-Alfvénic, i.e. when the ram pressure of the stellar wind dominates the magnetic pressure, a Jupiter-type magnetosphere with a bow shock, magnetopause, and magnetotail, is formed. At the same time, in the case of an extremely close orbital location of an exoplanet (e.g., $d<0.03$ AU for the Sun analogue star), where the stellar wind is still under acceleration and remains to be sub-magnetosonic and subAlfvénic (Ip et al., 2004; Preusse et al., 2005), an Alfvénic wing-type magnetosphere without a shock in the upstream region is formed. The character of the stellar wind impact on the planetary nearby plasma environment and inner atmosphere is different for the super- and sub- Alfvénic types of the magnetosphere and in each particular planet case it has to be properly taken into account. In the present paper, however, we do not consider the Alfvénic wing-type magnetospheres, aiming at moderately short orbit giant planets near solar-type stars, under the conditions of a super-Alfvénic stellar wind flow, i.e., with the magnetospheres having in a general case a bow shock, a magnetopause, a magnetotail, similar to the case of the solar system Jupiter.

\subsection{Magnetodisk - a key element of Hot Jupiter magnetosphere}

To explain an obvious survival and sufficient magnetospheric protection of close orbit Hot Jupiters under the extreme conditions of their host stars Khodachenko et al. (2012) 


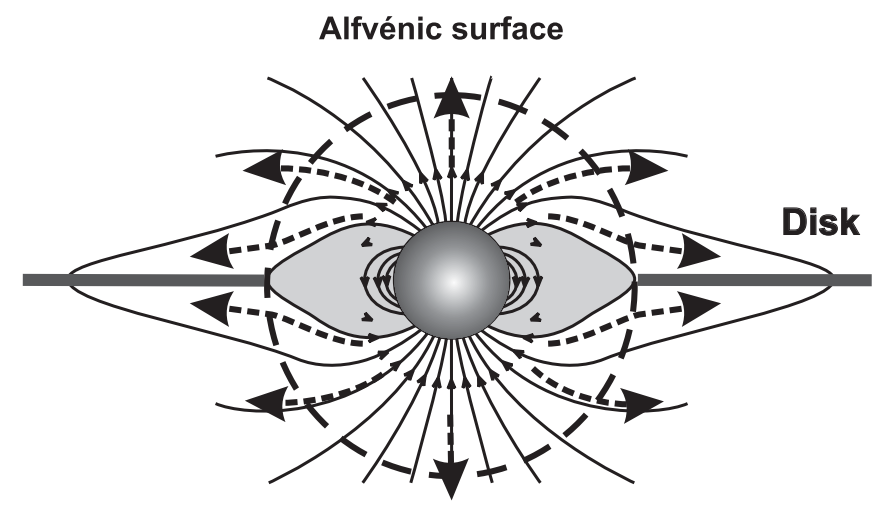

Figure 2. Schematic view of magnetodisk formation (adopted from Khodachenko et al. (2012).

proposed a more generic view of a Hot Jupiter magnetosphere. A key element in the proposed approach consists in taking into account of the upper atmosphere of a planet as an expanding dynamical gas layer heated and ionized by the stellar XUV radiation (Johansson et al., 2009; Koskinen et al., 2010, 2012). Interaction of the outflowing plasma with the rotating planetary magnetic dipole field leads to development of a current-carrying magnetodisk surrounding the exoplanet. The inner edge of magnetodisk is located at the so called Alfvénic surface $\left(r=R_{\mathrm{A}}\right)$ where the kinetic energy density of the moving plasma becomes equal to the energy density of the planetary magnetic field. This condition is equivalent also to the equality of the plasma ram pressure and magnetic pressure, or the Alfvén Mach number $M_{\mathrm{A}}^{2}=1$. Beyond the Alfvénic surface the expanding plasma is not guided any more by the dipole magnetic field. It deforms the field lines leading to creation of a current-carrying magnetodisk which in turn entirely changes the topology of planetary middle and outer magnetosphere.

According to Khodachenko et al. (2012), a Hot Jupiter's magnetodisk can be formed by different mechanisms, acting simultaneously: 1) the thermal expansion of the escaping planetary plasma wind, heated by the stellar radiation, and 2) the centrifugal acceleration of plasma by rotating planetary magnetic field in the co-rotation region, with subsequent release of material in the vicinity of the Alfvénic surface (so called "sling" mechanism). A self-consistent description of both these mechanisms represents an important and complex physical problem. So far only a qualitative insight into origin and interconnection of the inner (dipole dominated) and outer (magnetodisk-dominated) parts of the magnetosphere of a Hot Jupiter was suggested in Khodachenko et al. (2012). Two major regions with the different topology of magnetic field can be distinguished in the magnetosphere of a Hot Jupiter driven by the escaping plasma flow (Mestel, 1968). The first region corresponds to the inner magnetosphere, or so-called "dead zone", filled with closed dipole-type magnetic field lines. The magnetic field in the "dead zone" is strong enough to keep plasma locked with the planet. In the second region, so-called "wind zone", the expanding plasma drags and opens the magnetic field lines. These two regions are separated by Alfvénic surface $r=R_{\mathrm{A}}$ (see Figure 2). The plasma escaping along field lines beyond the Alfvénic surface not only deforms and stretches the original planetary dipole field, but also creates a thin disk-type current sheet in the equatorial region. Altogether, this leads to development of a new type of magnetodisk-dominated magnetosphere of a Hot Jupiter, which has no analogues among the solar system planets (Khodachenko et al., 2012). 


\subsection{Scaling of a magnetosphere with magnetodisk}

The proposed by Khodachenko et al. (2012)more complete view of the Hot Jupiter magnetosphere structure is based on the Paraboloid Magnetospheric Model (PMM). PMM is a semi-analytical approach to the modeling of planetary magnetosphere structure (Alexeev et al., 2003; Alexeev and Belenkaya, 2005; Alexeev et al., 2006; Khodachenko et al., 2012). The name of the model is derived from its key simplifying assumption that the magnetopause of a planet may be represented by a paraboloid surface co-axial with the direction of the ambient stellar wind plasma. The PMM calculates the magnetic field generated by a variety of current systems located on the boundaries and within the boundaries of a planetary magnetosphere. Besides of the intrinsic planetary magnetic dipole and magnetopause currents, the PMM has, among the main sources of magnetic field, also the electric current system of the magnetotail, and the induced ring currents of the magnetodisk. The model works without any restrictions imposed on the values of interplanetary medium parameters, enabling therefore the description of the whole variety of possible magnetosphere configurations caused by different intrinsic magnetic fields of exoplanets and various stellar wind conditions. As applied to the Hot Jupiters, PMM reveals that the electric currents induced in the plasma disk produce an essential effect on the overall magnetic field structure around the planet, resulting in the formation of a magnetodisk-dominated magnetosphere of a Hot Jupiter. Due to certain extension of the plasma disks around close-in exoplanets, the sizes of their magnetodisk-dominated magnetospheres are usually larger than those, followed from the traditional estimates with the equation (3.1), based on the account of only the screened planetary magnetic dipoles (Griemeier et al., 2004; Khodachenko et al., 2007a). In general, the role of magnetodisk may be attributed to an expansion of a part of the dipole magnetic flux from the inner magnetosphere regions outwards and a resulting increase of the magnetosphere size. The magnetic field produced by magnetodisk ring currents, dominates above the contribution of intrinsic magnetic dipole of a Hot Jupiter and finally determines the size and shape of the whole magnetosphere. Khodachenko et al. (2012) provided an approximate formula for estimation of the magnetopause stand-off distance taking into account the contribution of the magnetodisk:

$$
\frac{R_{\mathrm{s}}^{(d i p+M D)}}{r_{\mathrm{p}}} \sim \frac{B_{d 0 J}^{1 / 2}\left(1+\kappa^{2}\right)^{1 / 4}}{\left(2 \mu_{0} p_{\mathrm{sw}}\right)^{1 / 4}}\left(\frac{R_{\mathrm{AJ}}}{r_{\mathrm{p}}}\right)^{-1 / 2} \times\left(\frac{\omega_{\mathrm{p}}}{\omega_{\mathrm{J}}}\right)^{\frac{3 k+1}{10}}\left(\frac{\mathrm{d} M_{\mathrm{p}}^{(t h)} / \mathrm{d} t}{\mathrm{~d} M_{\mathrm{J}} / \mathrm{d} t}\right)^{\frac{1}{10}}
$$

where $R_{\mathrm{AJ}}, \frac{\mathrm{d} M_{\mathrm{J}}}{\mathrm{d} t}$, and $B_{\mathrm{d} 0 \mathrm{~J}}$ are the known values corresponding to the Alfvénic radius, mass load to the disk, and surface magnetic field for the solar system Jupiter. The coefficient $\kappa \approx 2.44$ is an amplifying factor of the inner magnetospheric field at the magnetopause (Alexeev et al., 2003), which is required to take into account the contribution of the Chapman-Ferraro field at the substellar point. It is connected with the form-factor $f_{0}$ from (3.1) as $\kappa=2 f_{0}$. Therefore, according to (4.1), for a given kinetic pressure of stellar wind, $p_{\mathrm{sw}}$, the size of magnetosphere increases for the increasing planetary angular velocity $\omega_{\mathrm{p}}$ and/or thermal mass loss rate $\mathrm{d} M_{\mathrm{p}}^{(t h)} / \mathrm{d} t$.

A slower, than the dipole-type decrease of magnetic field with distance comprises the essential specifics of the magnetodisk-dominated magnetospheres of Hot Jupiters. This results in their $40-70 \%$ larger scales, as compared to those traditionally estimated with taking into account of only the planetary dipole. Such larger magnetospheres, extending well beyond the planetary exosphere height, provide better protection of close-in planets against of the erosive action of extreme stellar winds (Khodachenko et al., 2007a). Table 2 summarizes the values for a Hot Jupiter magnetopause stand-off distance at different 
Table 2. Hot Jupiter Alfvénic radius, $R_{\mathrm{A}}$, and magnetopause stand-off distance for only a dipole controlled magnetosphere, $R_{\mathrm{s}}^{(d i p)}$, and a magnetosphere with magnetodisk, $R_{\mathrm{s}}^{(d i p+M D)}$, given by PMM. Full analog of the solar system Jupiter orbiting the Sun analog star at different orbits is considered. ${ }^{1}$ : Tidally locked. ${ }^{2}$ : Not tidally locked. ${ }^{3}$ : Jupiter

\begin{tabular}{|c|c|c|c|}
\hline $\begin{array}{l}d \\
{[\mathrm{AU}]}\end{array}$ & $\begin{array}{c}R_{s}^{(d i p+M D)} \\
{\left[r_{\mathrm{p}}\right]}\end{array}$ & $\begin{array}{c}R_{s}^{(d i p)} \\
{\left[r_{\mathrm{p}}\right]}\end{array}$ & $\begin{array}{l}R_{A} \\
{\left[r_{\mathrm{p}}\right]}\end{array}$ \\
\hline $0.045^{1}$ & 8.0 & 5.76 & 3.30 \\
\hline $0.1^{1}$ & 8.27 & 6.16 & 4.66 \\
\hline $0.3^{2}$ & 24.2 & 15.0 & 7.30 \\
\hline $5.2^{3}$ & 71.9 & 41.8 & 19.8 \\
\hline
\end{tabular}
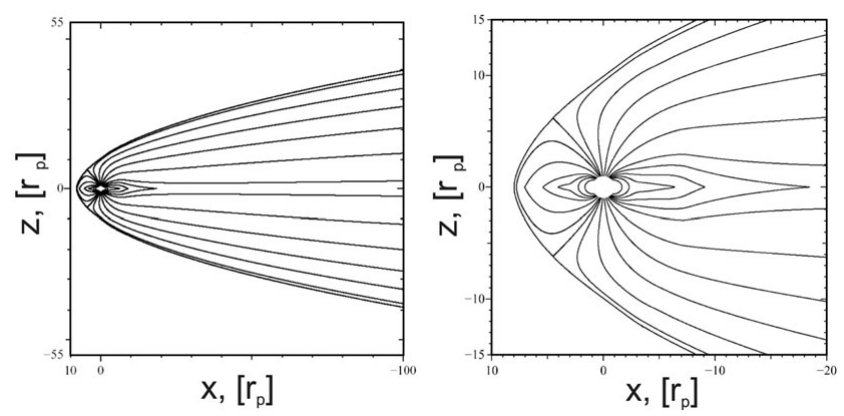

Figure 3. Typical view of a magnetodisk-dominated magnetosphere.

orbits around a Sun full analogue star and gives for the comparison the stand-off distance values, obtained with equation (3.1), i.e. in the case when the contribution of magnetodisk is ignored (e.g., a pure dipole case).

A typical example of the magnetic field structure in the in the magnetosphere of a Hot Jupiter, obtained with PMM, is shown in Figure 3.

\section{Conclusions}

To summarize this paper we would like to emphasize that stellar XUV radiation and stellar wind plasma flow strongly impact the environments of close-orbit exoplanets. Given the fact that the complete or partial tidal locking of such short periodic exoplanets may lead to relatively weak intrinsic planetary magnetic moments, the encountering stellar wind and CMEs will push the planetary magnetospheres down to the heights at which the ionization and pick-up of the planetary neutral atmosphere by the stellar plasma flow takes place. This makes the stellar activity and planetary magnetospheric protection to play a crucial role for the whole complex of planetary evolution processes, including atmosphere erosion and mass loss. Large enough extended magnetospheres are needed to protect the upper atmospheric environments against of stellar XUV and stellar wind/CMEs impacts.

The expanding and escaping upper atmospheric gas heated and ionized by the stellar radiation contributes to the build-up of the magnetodisk around the planet, which constitutes the major specifics of a Hot Jupiter magnetosphere considered in this work. The magnetic field produced by magnetodisk ring currents, dominates above the contribution of intrinsic magnetic dipole of a Hot Jupiter and finally determines the size and shape 
of the whole magnetosphere. A more realistic structure of the magnitodisk-dominated magnetosphere of a Hot Jupiter predicted by the Paraboloid Magnetospheric Model and its significantly larger size, as compared to a dipole-type magnetosphere, have important consequences for the study of magnetospheric protection of close orbit exoplanets.

\section{Acknowledgements}

This work was supported by the Austrian Science Foundation (FWF) (projects S11606N16 and P25587-N27). The authors acknowledge the EU FP7 project IMPEx for support of numerical modelling work and providing collaborative environment for research and communication.

\section{References}

Alexeev, I. I., Belenkaya, E. S., Bobrovnikov, S. Yu., \& Kalegaev, V. V. 2003, Space Sci.Rev., 107,7

Alexeev, I. I., Kalegaev, V. V., Belenkaya, E. S., Bobrovnikov, S. Yu., Bunce, E. J., Cowley, S. W. H., \& Nichols, J. D. 2006, Geophys.Res.Lett., 33, L08101

Alexeev, I. I., \& Belenkaya, E. S. 2005, Ann. Geophys., 23, 809

Audard, M., Güdel, M., Drake, J. J., \& Kashyap, V. L. 2000, ApJ, 541, 396

Ayres, T. R. 1997, JGR, 102, 1641

Erkaev, N. V., Penz, T., Lammer, H., Lichtenegger, H. I. M., Biernat, H. K., Wurz, P., Grießmeier, J.-M., \& Weiss, W. W. 2005, ApJ. Suppl. Ser., 157, 396

Erkaev, N. V., Lammer, H., Odert, P., Kulikov, Yu. N., Kislyakova, K. G., Khodachenko, M. L., Güdel, M., Hanslmeier, A., \& Biernat, H. 2013, Astrobio., (in press)

Gershberg, R. E. 2005, A Solar-Type Activity in Main-Sequence Stars, (Berlin,Heidelberg,New York: Springer)

Grießmeier, J.-M., Stadelmann, A., Penz, T., Lammer, H., Selsis, F., Ribas, I., Guinan, E. F., Motschmann, U., Biernat, H. K., \& Weiss, W. W. 2004, A $\&$ A, 425, 753

Grießmeier, J.-M., Preusse, S., Khodachenko, M. L., Motschmann, U., Mann, G., \& Rucker, H. O. 2007, Planet. \& Space Sci., 55, 618

Ip, W.-H., Kopp, A., \& Hu, J.-H. 2004, Astrophys. J., 602, L53

Johansson, E. P. G., Bagdonat, T., \& Motschmann, U. 2009, A \& A, 496, 869

Khodachenko, M. L., Ribas, I., Lammer, H., Grießmeier, J.-M., Leitner, M., Selsis, F., Eiroa, C., Hanslmeier, A., Biernat, H., Farrugia, C. J., \& Rucker, H. 2007a, Astrobio., 7, 167

Khodachenko, M. L., Lammer, H., Lichtenegger, H. I. M., Langmayr, D., Erkaev, N. V., Grießmeier, J.-M., Leitner, M., Penz, T., Biernat, H. K., Motschmann, U., \& Rucker, H. O. 2007b, Planet.Space Sci., 55, 631

Khodachenko, M. L., Alexeev, I. I., Belenkaya, E., Leitzinger, M., Odert, P., Grießmeier, J.-M., Zaqarashvili, T. V., Lammer, H., \& Rucker, H. O. 2012, ApJ, 744, 70

Koskinen, T., Yelle, R. V., Lavvas, P., \& Lewis, N. K. 2010, ApJ, 723, 116

Koskinen, T. T., Harris, M. J., Yelle, R. V., \& Lavvas, P. 2012, Icarus, (in press), http:// arXiv:1210.1535.

Lammer, H., Kasting, J. F., Chassefière, E., Johnson, R. E., Kulikov, Yu. N., \& Tian, F. 2008, Space Sci Rev., 139, 399

Lammer, H., Odert, P., Leitzinger, M., Khodachenko, M. L., Panchenko, M., Kulikov, Yu. N., Zhang, T. L., Lichtenegger, H. I. M., Erkaev, N. V., Wuchterl, G., Micela, G., Penz, A., Biernat, H. K., Weingrill, J., Steller, M., Ottacher, H., Hasiba, J., \& Hanslmeier, A. 2009,) A \& $A, 506,399$

Lichtenegger, H. I. M., Gröller, H., Lammer, H., Kulikov, Yu. N., \& Shematovich, V. 2009, Geophys. Res. Lett. ,36, CiteID L10204

Mestel, L. 1968, MNRAS, 138, 359

Newkirk, G., Jr. 1980, Geochim. Cosmochim. Acta Suppl., 13, 293

Parker, E. N. 1958, ApJ, 128, 664 
Penz, T., Erkaev, N. V., Kulikov, Yu. N., Langmayr, D., Lammer, H., Micela, G., CecchiPestellini, C., Biernat, H. K., Selsis, F., Barge, P., Deleuil M., \& Léger, A. 2008, Planet. Space Sci., 56, 1260

Preusse, S., Kopp, A., Büchner, J., \& Motschmann, U. 2005, A \& A, 434, 1191

Ribas, I., Guinan, E. F., Güdel, M., \& Audard, M. 2005, ApJ, 622, 680

Scalo, J., Kaltenegger, L., Segura, A. G., Fridlund, M., Ribas, I., Kulikov, Yu. N., Grenfell, J. L., Rauer, H., Odert, P., Leitzinger, M., Selsis, F., Khodachenko, M. L., Eiroa, C., Kasting, J., \& Lammer, H. 2007, Astrobiol., 7, 85

Sheeley, N. R., Jr., Wang, Y.-M., Hawley, S. H., Brueckner, G. E., Dere, K. P., Howard, R. A., Koomen, M. J., Korendyke, C. M., Michels, D. J., Paswaters, S. E., Socker, D. G., St. Cyr, O. C., Wang, D., Lamy, P. L., Llebaria, A., Schwenn, R., Simnett, G. M., Plunkett, S., \& Biesecker, D. A. 1997, ApJ, 484, 472

Skumanich, A. 1972, ApJ, 171, 565

Tian, F., Kasting, J. F., Liu, H., \& Roble, R. G. 2008, J. Geophys. Res., 113, E05008

Walker, J. C. G., Hays, P. B., \& Kasting, J. F. 1981, J. Geophys. Res., 86, 9776

Wood, B. E., Müller, H.-R., Zank, G. P., \& Linsky, J. L. 2002, ApJ, 574, 412

Wood, B. E., Müller, H. -R., Zank, G. P., Linsky, J. L., \& Redfield, S. 2005, ApJ, 628, L143 